\title{
SIMULTANEAOUS APPROXIMATION OF A FAMILY OF (STOCHASTIC) DIFFERENTIAL EQUATIONS
}

\author{
PHILTPPE CARMONA AND LAURE COUTIN
}

\begin{abstract}
To approximate the fractional integral of order a in $(0,1)$, we use an integral representation based on exponential functions introduced in a previous paper, and we present a scheme to approximate the whole family of associated linear differential equations:

$\mathrm{dy}(\mathrm{x}, \mathrm{t}) / \mathrm{dt}=\mathrm{u}-\mathrm{xy}(\mathrm{x}, \mathrm{t})$, for any $\mathrm{x}$ positive real.

We show how to extend these results to the stochastic case $u=$ "white noise", the fractional integration of which is a fractional brownian motion.
\end{abstract}

RÉsumé. En vue d'approximer l'intégrale fractionnaire d'ordre a compris entre 0 et 1 , nous utilisons une représentation intégrale à base d'exponentielles introduite dans un précédent papier, et présentons un schéma numérique permettant d'approximer simultanément la famille d'équations différentielles linéaires associées:

$\mathrm{dy}(\mathrm{x}, \mathrm{t}) / \mathrm{dt}=\mathrm{u}-\mathrm{xy}(\mathrm{x}, \mathrm{t})$, pour tout $\mathrm{x}$ reel positif.

Ensuite, nous montrons comment étendre ces résultats au cadre stochastique, $\mathbf{u}=$ "bruit blanc", pour lequel l'intégrale fractionnaire est un mouvement brownien fractionnaire.

\section{INTRODUCTION}

Suppose that you want to compute the fractional integral

$$
I_{0,+}^{\alpha} u(t)=\frac{1}{\Gamma(\alpha)} \int_{0}^{t}(t-s)^{\alpha-1} u(s) d s \quad(0<\alpha<1) .
$$

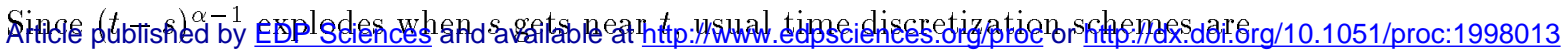
unsuitable. But if we combine the identity

$$
\frac{1}{z^{a}}=\frac{1}{\Gamma(a)} \int_{0}^{\infty} x^{a-1} e^{-z x} d x \quad(z>0, a>0),
$$

with Fubini's Theorem, we obtain, for $a=1-\alpha$, another representation of the fractional integral:

$$
I_{0,+}^{\alpha} u(t)=\frac{1}{\Gamma(\alpha) \Gamma(1-\alpha)} \int_{0}^{\infty} d x x^{-\alpha} \int_{0}^{t} e^{-x(t-s)} u(s) d s .
$$

More generally, one can consider the convolution integral

$$
I_{0,+}^{h} u(t)=\int_{0}^{t} h(t-s) u(s) d s
$$

where $h$ is the Laplace transform of a sigma-finite measure $\mu$

$$
h(s)=\int e^{-s x} \mu(d x) \quad(s>0),
$$

\footnotetext{
(C) Société de Mathématiques Appliquées et Industrielles. Typeset by $\mathrm{H}_{\mathrm{E}} \mathrm{X}$.

P. Carmona, L. Coutin : Laboratoire de Statistique et Probabilités, Université Paul Sabatier, 118 route de Narbonne, 31062 Toulouse Cedex 4. E-mail :carmona@cict.fr coutin@cict.fr .
} 
such that

$$
\forall t>0, \quad \int_{0}^{t} \int|u(s)| e^{-x(t-s)} \mu(d x)<+\infty,
$$

(if $\mu$ is a measure on $\mathbb{R}_{+}$and $u$ is locally bounded, it is enough that $\int(1 \wedge$ $\left.\left.x^{-1}\right) \mu(d x)<+\infty\right)$. Then, we have the representation of $I_{0,+}^{h} u$ as the mixture

$$
I_{0,+}^{h} u(t)=\int \mu(d x) y_{t}^{x}
$$

where $y^{x}$ is the solution of the ordinary (linear) differential equation

$$
\dot{y}^{x}=u-x y^{x}, \quad y^{x}(0)=0 .
$$

We are then naturally led to a two step approximation scheme.

The first step is the spatial approximation. The measure $\mu$ is approximated by a finite linear combination of Dirac masses

$$
\mu \simeq \mu^{\pi}=\sum_{x \in \pi} c_{x} \delta_{x},
$$

where $\pi$ is a finite subset. Thus,

$$
I_{0,+}^{h} u(t) \simeq I_{0,+}^{h, \pi} u(t)=\int \mu^{\pi}(d x) y_{t}^{x}=\sum_{\pi} c_{x} y_{t}^{x} .
$$

The second step is the time discretization. Each function $y^{x}, x \in \pi$, is approximated by a function ${ }^{(n)} y^{x}(t)$ depending on a partition $\Delta_{n}$ of $(0,+\infty)$.

This approach is successful when in the spatial aproximation we manage to keep the dimension low, that is the cardinal of $\pi$ small. It has been shown (see $[2,3]$ ), that even if we replace $u$ by a very rough signal, $u=\dot{B}=$ " white noise", which is the case when we need to approximate fractional Brownian motion, then to obtain a precision of the order $\epsilon$

$$
\sup _{0 \leq s \leq t}\left|I_{0,+}^{h} u(s)-I_{0,+}^{h, \pi} u(s)\right| \simeq \epsilon,
$$

one needed roughly $|\pi(\epsilon)| \simeq \frac{1}{\sqrt{\epsilon}} \log \left(\frac{1}{\sqrt{\epsilon}}\right)$ points.

\section{The time Discretization Schemes}

In this communication we shall focus our interest on the time discretization step. It is natural to use an Euler's scheme:

$$
\Delta y^{x}(t)=y^{x}(t+\Delta t)-y^{x}(t)=\left(u(t)-x y^{x}(t)\right) \Delta t .
$$

Keeping in mind the example of $u=\dot{B}=$ "white noise", we introduce the primitive of $u$,

$$
U(t)=\int_{0}^{t} u(s) d s,
$$

and we prefer to use the scheme

$$
\Delta y^{x}(t)=\Delta U(t)-x y^{x}(t) \Delta t
$$

To be more precise we consider a regular partition of the interval $[0, T]:\left(t_{k}=\right.$ $T k / n, 0 \leq k \leq n)$ and

$$
{ }^{(n)} y^{x}\left(t_{k+1}\right)={ }^{(n)} y^{x}\left(t_{k}\right)+\left(U\left(t_{k+1}\right)-U\left(t_{k}\right)\right)-x^{(n)} y^{x}\left(t_{k}\right)\left(t_{k+1}-t_{k}\right) .
$$

A classical Gronwall's type upper bound is (see, e.g. Bulirsch and Stoer [1], section $7.2)$

$$
\left|{ }^{(n)} y^{x}(t)-y^{x}(t)\right| \leq \frac{e^{x t}}{x t}(1+x) \sup \{|U(r)-U(s)|,|r-s| \leq 1 / n, 0 \leq r, s \leq t\} .
$$


This may not be a very good upper bound. Indeed, in the case of fractional Brownian motion, $U$ is Brownian motion, and therefore locally Hölder continuous of index $\gamma \in\left(0, \frac{1}{2}\right)$. The upper bound we obtain on the interval $[0,1]$ is, up to multiplicative constants, $e^{x} n^{-\gamma}$. We have to take into account that to obtain a precision of order $\epsilon$, one has to consider a partition $\pi(\epsilon)$ with end point $\sup (\pi(\epsilon)) \simeq \epsilon^{-\frac{2}{2 \alpha+3}}$; therefore, $n$ should be, very roughly, $n \simeq \exp \left(\epsilon^{-\frac{2}{2 \alpha+3}}\right)$ which is clearly inacceptable.

Hence, we have to build a scheme adapted to the approximation of the whole family of linear differential equations (1.1). More precisely, we let $h=T / n$ and define

$$
\begin{aligned}
& y^{x}(t)=e^{-x t} z^{x}(t), \quad z^{x}(t)=\int_{0}^{t} f^{x}(s) u(s) d s, \quad{ }^{(n)} y^{x}(t)=e^{-x t}(n) z^{x}(t), \\
& { }^{(n)} z^{x}(t)= \begin{cases}\int_{0}^{t} f_{n}^{x}(s) u(s) d s & \text { if } x h \leq \frac{1}{2} ; \\
0 & \text { otherwise. }\end{cases} \\
& f_{n}^{x}(s)=e^{x t_{k}} \quad \text { if } t_{k} \leq x<t_{k+1}, \quad f^{x}(s)=e^{x s} .
\end{aligned}
$$

Our main result for deterministic $u$ is the

Theorem 2.1. Assume that for a $p>1, u$ is in every $L^{p}(0, t), t>0$. Then

$$
\operatorname{supsup}_{x>0}\left|{ }_{t \leq T}^{(n)} y^{x}(t)-y^{x}(t)\right| \leq C(q, T) n^{-1 / q},
$$

where $q$ is the conjugate exponent of $p, 1=1 / p+1 / q$, and

$$
C(q, T)=(2 T / q)^{1 / q} \sup \left((q+1)^{-1 / q}, \frac{1}{2} e^{\frac{1}{2}}\right)\|u\|_{L^{p}(0, T)} .
$$

If we combine the spatial approximation for a geometric subdivision $\pi=\left\{r^{i}\right\}_{i}$ of $[1 / N, N]$ of geometric ratio $r>1$, with a time discretizationof step $1 / n$, we get, for fractional integration

Corollary 2.2. Assume that for a $p>1, u$ is in every $L^{p}(0, t), t>0$. Then,

$\left|I_{0,+}^{h} u(t)-I_{0,+}^{h, \pi, n} u(t)\right| \leq c\|u\|_{L^{p}(0, t)}\left(N^{-(1-\alpha)}+N^{-(\alpha+1+1 / q)}+(r-1)^{2}+n^{-1 / q} N^{1-\alpha}\right)$.

The proof of Theorem 2.1 relies heavily on the upper bounds given in the

LEMMA 2.3 .

$$
\begin{gathered}
\sup \left(\left|{ }^{(n)} z^{x}(t)\right|,\left|z^{x}(t)\right|\right) \leq\|u\|_{L^{p}(0, t)}\left(\frac{e^{q x t}-1}{q x}\right)^{1 / q} \\
\left|z^{x}(t)-{ }^{(n)} z^{x}(t)\right| \leq\|u\|_{L^{p}(0, t)}(x T / n) e^{x T / n}(q(q+1))^{-1 / q} x^{-1 / q} e^{x t} .
\end{gathered}
$$

When $U$ is not deterministic anymore, but a Brownian motion, this Lemma has a stochastic analog. Let

$$
\begin{array}{rlrl}
Y^{x}(t) & =e^{-x t} Z^{x}(t), & Z^{x}(t) & =\int_{0}^{t} f^{x}(s) d B_{s}, \\
{ }^{(n)} Y^{x}(t) & =e^{-x t}{ }^{(n)} Z^{x}(t), \quad{ }^{(n)} Z^{x}(t) & =\int_{0}^{t} f_{n}^{x}(s) d B_{s} .
\end{array}
$$

LeMma 2.4.

$$
\begin{gathered}
\sup \left(\left\|\sup _{s \leq t}\left|{ }^{(n)} Z^{x}(s)\right|\right\|_{2},\left\|\sup _{s \leq t}\left|Z^{x}(s)\right|\right\|_{2}\right) \leq 2\left(\frac{e^{2 x t}-1}{2 x}\right)^{\frac{1}{2}} . \\
\left\|\sup _{s \leq t}\left|{ }^{(n)} Z^{x}(s)-Z^{x}(s)\right|\right\|_{2} \leq \frac{2}{\sqrt{6}}(x T / n) e^{x T / n} x^{-\frac{1}{2}} e^{x t} .
\end{gathered}
$$


We cannot obtain a uniform result, as in the deterministic case, because in a stochastic framework, supremums don't behave well. More precisely you can have two random variables $X_{1}$ and $X_{2}$ such that $\left\|X_{i}\right\|_{2} \leq c$, but $\left\|\sup \left(X_{1}, X_{2}\right)\right\|_{2}>c$ (take Bernoulli random variables $X_{2}=1-X_{1}$ ). Therefore we have to loose something in the process of obtaining good upper bounds on the $Y$ 's based on upper bounds on the Z's.

Given a process $(U(t) ; t \geq 0)$ and $x>0$, we consider $V(t)=e^{-t} U(t)$, and the two quantities

$$
U^{*}(t)=\sup _{s \leq t}|U(s)|, \quad V^{*}(t)=\sup _{s \leq t}|V(s)| .
$$

Lemma 2.5. Assume that for a $p \in(1,+\infty)$, and a constant $C>0$, we have $\left\|U^{*}(t)\right\|_{p} \leq C e^{x t}$. Then,

$$
\left\|V^{*}(t)\right\|_{p} \leq C(1+x t) e .
$$

From Lemmae 2.4 and 2.5 we immediately deduce the

THEOREM 2.6. For the stochastic scheme (2.3), we have the upper bounds:

$$
\begin{gathered}
\sup \left(\left\|\sup _{s \leq t}\left|{ }^{(n)} Y^{x}(s)\right|\right\|_{2},\left\|\sup _{s \leq t}\left|Y^{x}(s)\right|\right\|_{2}\right) \leq \sqrt{\frac{2}{x}}(1+x t) . \\
\left\|\sup _{s \leq t}\left|{ }^{(n)} Y^{x}(s)-Y^{x}(s)\right|\right\|_{2} \leq \frac{2 e}{\sqrt{6}}(x T / n) e^{x t / n} x^{-\frac{1}{2}}(1+x t) .
\end{gathered}
$$

\section{ProOfs}

Proof of Lemma 2.5. Without loss in generality, we may assume $x=1$. For every integer $n \geq 1$, we have

$$
\begin{aligned}
\left\|V^{*}(t)\right\|_{p} & =\left\|\sup _{0 \leq i<n}\left(\sup _{i t / n \leq s \leq(i+1) t / n} e^{-s}|U(s)|\right)\right\|_{p} \\
& \leq\left\|\sum_{0 \leq i<n} \sup _{i t / n \leq s \leq(i+1) t / n} e^{-s}|U(s)|\right\|_{p} \\
& \leq \sum_{0 \leq i<n}\left\|\sup _{i t / n \leq s \leq(i+1) t / n} e^{-s}|U(s)|\right\|_{p} \\
& \leq \sum_{0 \leq i<n} e^{-i t / n}\left\|U^{*}((i+1) t / n)\right\|_{p} \\
\leq & \sum_{0 \leq i<n} e^{-i t / n} C e^{(i+1) t / n}=C n e^{t / n} \\
& \leq e(1+t) C
\end{aligned}
$$

by taking $n=1+\lfloor t\rfloor$.

Proof of Lemma 2.4. Since $\left(Z^{x}(t) ; t \geq 0\right)$ is a square integrable martingale, Doob's $L^{2}$ inequality implies that

$$
\left\|\sup _{s \leq t}\left|Z^{x}(s)\right|\right\|_{2} \leq 2\left\|Z^{x}(t)\right\|_{2}=2\left\|f^{x}\right\|_{L^{2}(0, t)}=2\left(\frac{e^{2 x t}-1}{2 x}\right)^{\frac{1}{2}} .
$$


Similarly,

$$
\begin{aligned}
\left\|\sup _{s \leq t}\left|{ }^{(n)} Z^{x}(s)\right|\right\|_{2} & \leq 2\left\|(n) Z^{x}(t)\right\|_{2}=2\left\|f_{n}^{x}\right\|_{L^{2}(0, t)} \\
& =2\left(\sum \int_{t_{k} \wedge t}^{t_{k+1} \wedge t} e^{2 x t_{k}} d s\right)^{\frac{1}{2}} \leq 2\left\|f^{x}\right\|_{L^{2}(0, t)}=2\left(\frac{e^{2 x t}-1}{2 x}\right)^{\frac{1}{2}} .
\end{aligned}
$$

and

$$
\begin{aligned}
\left\|\sup _{s \leq t} \mid{ }^{(n)} Z^{x}(s)-Z^{x}(s)\right\|_{2}^{2} & \leq 4\|\|{ }^{(n)} Z^{x}(t)-Z^{x}(t)\|\|_{2}^{2} \\
& =4\left\|f^{x}-f_{n}^{x}\right\|_{L^{2}(0, t)}^{2}=4 \sum \int_{t_{k} \wedge t}^{t_{k+1} \wedge t}\left(e^{x s}-e^{x t_{k}}\right)^{2} d s
\end{aligned}
$$

Taking into account that for $0 \leq a \leq b, 0 \leq e^{b}-e^{a} \leq(b-a) e^{b}$, we have

$$
\begin{aligned}
\left\|\sup _{s \leq t} \mid{ }^{(n)} Z^{x}(s)-Z^{x}(s)\right\|_{2}^{2} & \leq 4 \sum \frac{1}{3}\left(t_{k} \wedge t-t_{k+1} \wedge t\right)^{3} e^{2 x\left(t_{k+1} \wedge t\right)} x^{2} \\
& \leq(4 / 3)(x h)^{2} \int_{0}^{t+h} e^{2 x s} d s \\
& \leq(4 / 6))(x h)^{2} e^{2 x h} x^{-1} e^{2 x t} .
\end{aligned}
$$

Proof of Lemma 2.3. By Hölder's inequality,

$$
\begin{aligned}
\left|z^{x}(t)\right| & \leq\|u\|_{L^{p}(0, t)} \mid f^{x} \|_{L^{q}(0, t)} \\
& =\|u\|_{L^{p}(0, t)}\left(\frac{e^{q x t}-1}{q x}\right)^{1 / q} .
\end{aligned}
$$

Similarly,

$$
\left|{ }^{(n)} z^{x}(t)\right| \leq\|u\|_{L^{p}(0, t)}\left\|f_{n}^{x}\right\|_{L^{q}(0, t)}
$$

and

$$
\begin{aligned}
\int_{0}^{t} f_{n}^{x}(s)^{q} d s & =\sum \int_{t_{k} \wedge t}^{t_{k+1} \wedge t} e^{x t_{k}} d s \\
& \leq \sum \int_{t_{k} \wedge t}^{t_{k+1} \wedge t} e^{x s} d s \quad=\frac{e^{q x t}-1}{q x} .
\end{aligned}
$$

Observe that, since if $0 \leq a \leq b, 0 \leq e^{b}-e^{a} \leq(b-a) e^{b}$, if $x h \leq \frac{1}{2}$, then

$$
\begin{aligned}
\left\|f_{x}-f_{n}^{x}\right\|_{L^{q}(0, t)}^{q} & =\sum \int_{t_{k} \wedge t}^{t_{k+1} \wedge t}\left(e^{x s}-e^{x t_{k}}\right)^{q} d s \\
& \leq \sum \int_{t_{k} \wedge t}^{t_{k+1} \wedge t}\left(s-t_{k}\right)^{q} x^{q} e^{q x t_{k+1}} d s \\
& \leq \frac{1}{q+1} \sum\left(t_{k+1} \wedge t-t_{k} \wedge t\right)^{q+1} x^{q} e^{q x t_{k+1}} \\
& \leq \frac{1}{q+1} x^{q} h^{q} \frac{\exp (q x(t+h))-1}{q x}
\end{aligned}
$$


Therefore

$$
\begin{aligned}
\left|{ }^{(n)} z^{x}(t)-z^{x}(t)\right| & \leq\|u\|_{L^{p}(0, t)}\left\|f_{n}^{x} f^{x}\right\|_{L^{q}(0, t)} \\
& \leq\|u\|_{L^{p}(0, t)}(q(q+1))^{-1 / q}(x h) e^{x h} x^{-1 / q} e^{x t} \\
& \leq\|u\|_{L^{p}(0, t)}(q(q+1))^{-1 / q} \frac{1}{2} e^{\frac{1}{2}}(2 T)^{1 / q} n^{-1 / q} e^{x t}
\end{aligned}
$$

Proof of Theorem 2.1. From Lemma 2.3 we obtain, if $x h \leq \frac{1}{2}$,

$$
\left|{ }^{(n)} z^{x}(t)-z^{x}(t)\right| \leq\|u\|_{L^{p}(0, t)}(q(q+1))^{-1 / q} \frac{1}{2} e^{\frac{1}{2}}(2 T)^{1 / q} n^{-1 / q} .
$$

And, if $x h>\frac{1}{2}$,

$$
\left|{ }^{(n)} z^{x}(t)-z^{x}(t)\right|=\left|z^{x}(t)\right| \leq\|u\|_{L^{p}(0, t)}(q x)^{-1 / q} \leq\|u\|_{L^{p}(0, t)}(2 T / q)^{1 / q} n^{-1 / q}
$$

\section{REFERENCES}

[1] R. BuliRsch and J. Stoen, Introduction to numerical analysis, Springer-Verlag, 1980.

[2] Ph. Carmona, L. Coutin, and G. Montseny, Applications of a representation of long memory Gaussian processes, Submitted to Stochastic Processes and their Applications, June 1997.

[3] - A diffusive Markovian representation of fractional Brownian motion with Hurst parameter less than $1 / 2$, To be published, June 1997. 